\title{
WHY THE LATEST ANTENATAL HIV PREVALENCE SURVEY RESULTS ARE STILL BAD NEWS
}

Clive Evian, $M B$ BCh, MMed (Community Health)

AIDS Management and Support/Lifeworks, Hon. Lecturer, Department of Community Health, University of the Witruatersrand

After the Minister of Health delayed releasing the 2002 antenatal HIV survey to the South African public for many months, the report on the 2002 antenatal survey results' was somewhat misleading in giving the impression to the public that the epidemic is 'stabilising' and coming under control. Is this spin another example of the national denial recently so well propagated by President Mbeki ('Personally I do not know anyone who has died of AIDS')?

If the essential dynamics of the epidemic are understood I think they may lead to a different conclusion, and this issue needs more exploration and expert opinion. It will be seen that in actual fact the recent HIV antenatal surveillance survey shows that the epidemic is clearly still very much out of control (at the very least among women in the pregnancy age group). The charts and data below show the overall HIV prevalence in pregnant women from 1990 to 2002 (Fig. 1) and the age-specific HIV prevalence from 2000 - 2002 (Fig. 2).

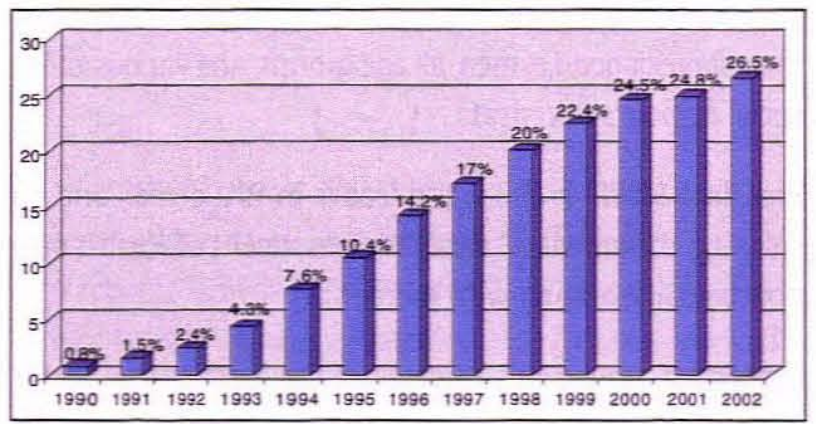

Fig. 1. HIV prevalence among pregnant women in the public health services in South Africa, 1990 - 2002.

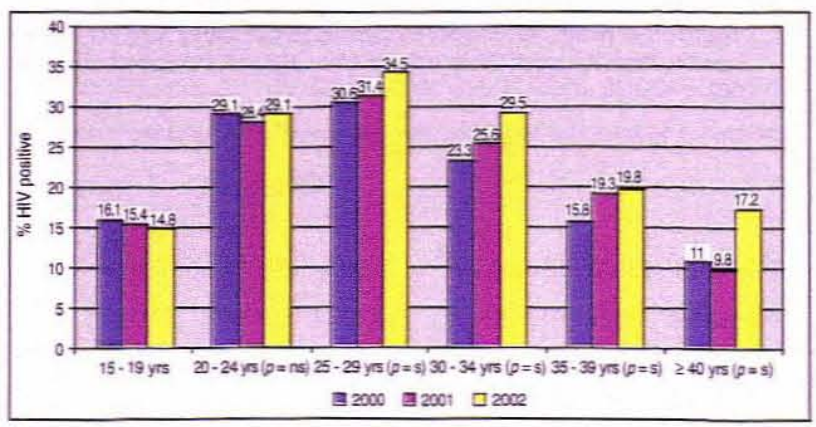

Fig. 2. Age-specific HIV prevalence among pregnant women in the public health services in South Africa, 2000 - 2002.

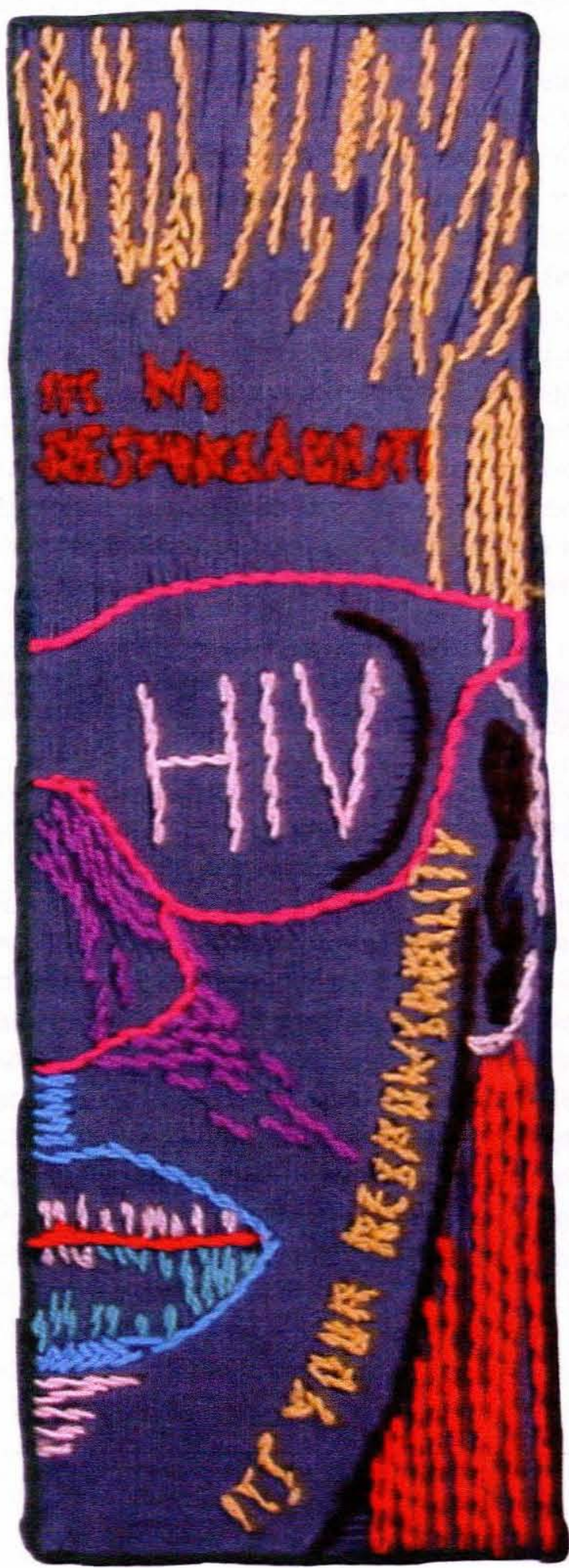

NOVEMAER 2003 
When the epidemic began in the late 1980s (see Fig. 1), almost $100 \%$ of the population was HIV-negative. The epidemic took a few years to establish itself, because HIV is a poorly transmitted virus and it therefore took some time to build up a critical pool of infected people (i.e 1989 1992). Once the pool was large enough, i.e. $1-2 \%$ of sexually active adults (extrapolating to many tens of thousands of people), the epidemic started to grow exponentially (1993 - 1997). At this stage each new infection added one more to the prevalence and the rate of increase was very high (1992 - 1998). After some time (because HIV is a long chronic infection), many people are repeatedly reinfected; these reinfections do not add to the prevalence, and the epidemic appears to slow down (1998 - 2001). In addition, at this more mature phase of the epidemic, most very vulnerable people have now acquired HIV (except for the youngest age bands), and a 'saturation' of a sort may be reached. Also people have started dying from the disease ( 7 - 10 years after infection), and if the number of deaths equals the number of new infections, the prevalence will remain the same (you cannot contribute to prevalence if you are no longer around to be tested). Clearly the epidemic is not under control yet. For us to claim control, the HIV prevalence must be on the decline and decline consistently over a number of years. Not only is the prevalence not declining, but it is inclining (2001 - 2002). While this incline may not be statistically significant, there is a very strong hint of a rise. Any continued rise in the presence of a maturing epidemic is very bad news.

Even more serious than the evidence from the overall epidemic are the facts arising out of the age-specific HIV prevalence among pregnant women (see Fig. 2).

Firstly, if one looks at the prevalence in the 15 - 19-year age group, it is falling slightly. However, the mere fact that approximately $15 \%$ of teenage girls are HIV-infected is terrible. It means that in the space of about $2-3$ years teenage girls are going from almost zero prevalence (in the 10 - 14-year age group) to a striking $15 \%$. This is alarming even if the figure is decreasing slightly. A high proportion of these infections must be arising out of sexual contact with older men (from whom the infection must originate). Also, the age band most sensitive to change is the 15 19 -year band (prevalence in older age bands will take longer to show declining trends over time owing to the chronic nature of HIV infection), as young people enter the sexually active phase of their lives. If the prevalence in this band is indeed being maintained at high levels it is very bad news.

Now, to go one step further, note how steep the increase in HIV infection is between the 15 - 19 age band and the 20 24 band - almost a doubling of the prevalence, i.e approximately a $100 \%$ increase. This indicates not only that are prevention strategies failing, but they are failing with distinction. This upward trend is being carried through to the 25 - 29- and 30 - 34-year age bands as well, where there has been a significant increase in HIV prevalence from 2001 to 2002.

The above data are revealing concerning both the upward trend in prevalence in the 20 - 34-year bands and the fact that this is taking place in a time period where the epidemic is maturing. The rate of new infections, or of people being reinfected, may be as bad now as it was $5-6$ years ago lassuming that there is also an attrition of HIV-positive people through death).

The 101 of HIV prevention is to face up to the reality of the epidemic. This cannot be achieved if the Department of Health is not fully informed and fully understanding of the true dynamic of the epidemic and its implications. And it cannot be achieved if the public are not fully informed of the implications of the results of such an important survey.

The latest antenatal data are indeed very worrying and suggest that the national strategy (if it exists) is failing. Failure is one thing, and it may be inevitable, but not to recognise it is a possibly even more worrying failure. It is also high time that the Ministry of Health expands its HIV surveillance to include more than just pregnant women in their surveillance, i.e. men, all age groups, and various other social demographic strata.

President Mbeki is the world leader in HIV denial, and by spinning the antenatal data the Department of Health may now be collaborating with this denial.

Leadership around this epidemic is the most critical factor in the true stabilisation of the epidemic in some countries north of South Africa. When will the leadership of this country wake up? If not now - when?

1. Department of Health. National HIV and Syphilis Seroprevalence Survey of Women Attending Public Antenatal Clinies in South Africa. Pretoria: Department of Health, 2002. 